\title{
On Einstein's Program and Quantum Double Slit Experiment
}

\author{
Claude Elbaz \\ Correspondence: Claude Elbaz, Academie Europeenne Interdisciplinaire de Science (A.E.I.S.), Paris, France
}

Received: June 22, 2016 Accepted: July 7, $2016 \quad$ Online Published: July 12, 2016

doi:10.11114/set.v3i1.1730

URL: http://dx.doi.org/10.11114/set.v3i1.1730

\begin{abstract}
The Einstein's program enables a theoretical economy for quantum double slit experiment, in its wave-particle duality behavior, with the unification of first and second quantifications for light and matter. It introduces a space-like amplitude function $\mathrm{u}(\mathrm{r}, \mathrm{t})$, which completes the usual time-like functions $\psi(\mathrm{r}, \mathrm{t})$ of quantum mechanics and quantum fields. The Einstein's program is founded upon a scalar field propagating at speed of light c. It forms a common relativist framework, for classical and quantum properties of matter and interactions. Matter properties derive from standing waves, and interactions from progressive waves. The classical domain arises in the geometrical optics approximation, when frequencies are infinitely high, and then hidden. The quantum domain corresponds to wave optics approximation. Adiabatic variations of frequencies yield electromagnetic interaction and dynamical laws of energy-momentum conservation and least action principle.
\end{abstract}

Keywords: Einstein's program, quantum mechanics, hidden variables, wave-particle duality, double-slit experiment

\section{Introduction}

The double-slit experiment plays an emblematic role in quantum mechanics, since it exhibits many of its main specific characters: the wave-particle duality, the wave diffraction by slits, the classical Fourier conditions corresponding to the Heisenberg quantum relations, and the probabilistic manifestation of a particle in recordings. These quantum features had much mobilized physicists, especially after 1920 years. Einstein remained resolutely opposed to the probabilistic orientation, generally adopted by physicists, which led to the development of quantum mechanics, even though, or because, he brought some major contributions in the elaboration of quantum physics, like the discovery of the particle behavior for light in photoelectric effect in 1905, introducing then the first quantum particle, for which he was awarded the Nobel prize in 1921, or the stimulated emission for light in 1917, at the base of lasers conceptions.

He asserted that, in spite of its formal and experimental successes, «The statistical character of the present theory would then have to be a necessary consequence of the incompleteness of the description of the systems in quantum mechanics... Above all, however, the reader should be convinced that I fully recognize the very important progress which the statistical quantum theory has brought in physics... this theory is until now the only one which unites the corpuscular and undulatory dual character of matter in a logically satisfactory fashion; and the (testable) relations, which are contained in it, are, within the natural limits fixed by the undeterminacy-relation, complete. The formal relations which are given in this theory-i.e., its entire mathematical formalism-will probably have to be maintained, in the form of logical inferences, in every useful future theory" (Einstein A., 1949).

We notice that, in absence of a suitable pictorial model for the quantum wave-particle duality, priority was given to abstract mathematical formalism. It leads to problems for physical representation, even for prominent physicists like R. Feynman. He recognized that "unlike ordinary experience, it is very difficult to get used to, and it appears peculiar and mysterious for everyone- both to the novice and to the experienced physicist. Even the experts do not understand it the way they would like to, and it is perfectly reasonable that they should not, because all of direct, human experience and human intuition applies to large objects. We know how large objects will act, but things on a small scale just do not act that way. So we have to learn about them in a sort of abstract or imaginative fashion and not by connection with our direct experience." The double-slit experiment exhibits "a phenomenon which is impossible, absolutely impossible, to explain in any classical way, and which has in it the heart of quantum mechanics. In reality, it contains the only mystery" (Feynman, 1965).

On the contrary, Einstein gave priority to physical models for representations. At first sight, this looks like paradoxical on his part, since, from the beginning, the relativity theory has been commonly considered as incomprehensible, both for its abstract mathematical formalism, and for its unusual physical manifestations. Nevertheless, it is well known that Einstein 
was accustomed to rely upon thoughts experiments which describe physical experiments, like the reciprocal electro dynamic action of a magnet and a conductor, at the base of special relativity, or the motion of bodies in free fall, for the elaboration of general relativity and the geometry of space. He asserted that "Most of the fundamental ideas of science are essentially simple, and may, as a rule, be expressed in a language comprehensible to everyone. To follow up these ideas demands the knowledge of a highly refined technique of investigation. Mathematics as a tool of reasoning is necessary if we wish to draw conclusions which may be compared with experiment. So long as we are concerned only with fundamental physical ideas, we may avoid the language of mathematics" (Einstein \& Infeld, 1938).

The basic models of waves and particles are physically and mathematically opposed and complementary: the waves are extended through space while the particles are concentrated. The concept of material particle is characterized by a double discontinuity, in space and in time. In space, by delimiting an inner «full» part, and an outer « empty » part. In time, by delimiting a previous time, defined before its creation, and a posterior time after, during which it is present. The concept of wave implies a continue dependence upon time, either infinite for a plane wave, or limited for a wave packet. Consequently, in classical physics, either one model, or the other, was admitted as more fundamental for the basic constitution of the universe. In this context, the double-slit experiment, performed originally by Thomas Young in 1801, played a vital part in the acceptance of the wave theory of light, to replace the particle theory adopted following Newton.

When Einstein reintroduced the particle behavior for light, he preserved its theoretical and experimental wave characters. He used cautiously the term heuristic, in the title of his article: Concerning an Heuristic Point of View Toward the Emission and Transformation of Light, (Einstein, 1905). Then, in spite of the particle signification attributed to its energy, through the Planck's relation $\mathrm{E}=\mathrm{h} v$, physicists continued to admit that light propagated as a wave. For instance, in the Bohr's model of atoms, which was a cornerstone in the development of quantum mechanics, an electron, as a mass-point, moved along a trajectory around a nucleus, while exchanging electromagnetic waves with it. The non relativist Schrödinger equation, enabled to supersede this model. Mathematically, through a second order partial differential equation with respect to space, it describes a wave, independently of its physical nature. A single particle is implicitly described by the first order partial differential equation with respect to time, linked to its energy. It restricts to the kinetic energy $E_{k}=p^{2} / 2 m$, instead of the main energy $E=m^{2} \approx E_{k}+E_{0}$. This emphasizes that the rest energy $\mathrm{E}_{0}=\mathrm{m}_{0} \mathrm{c}^{2}$, is admitted as remaining constant in whole processes involved afterwards, justifying that it was then eluded, or hidden. Consequently, it is admitted that the mass $\mathrm{m}_{0}$ of a particle, like an electron, remains unaffected by its motion and by its interactions. Like in classical physics, the mass-energy extended outside the point-particle in neglected, and hidden. Nevertheless, its underlying action continues to operate in that case, but as a second order wave approximation, as a perturbation, which corresponds to the Heisenberg relations of quantum mechanics, linked to the probabilistic position of a single particle (Elbaz 2015).

Nowadays, in extension of quantum mechanics, the standard model forms a consistent system to describe universe. It admits that the whole universe is constituted of fundamental particles, both for matter and for three, out of the four known, different kinds of interactions. They all behave either as waves or as particles, in a probabilistic framework. The fourth interaction, gravitation, has resisted to its quantification since a century. It is still described by general relativity, through a continuous field, in a classical and determinist framework.

In addition to its only partial description of the universe, the standard model differs from, and goes beyond, quantum mechanics, since it leans on relativist quantum fields. They no longer restrict to a single particle, but apply to many identical particles, each one being created or annihilated inside the corresponding field. The two basic categories of quantum particles, the bosons and the fermions, differ from one another by their statistical properties, which are then in relation with sets of identical particles: Bose-Einstein statistic laws for the first ones, and Fermi-Dirac for the second. A single particle appears only as a particular manifestation of a more fundamental continuous field, expressed mathematically by partial derivative equations.

In extension of general relativity and of his different discoveries, including in quantum physics, Einstein had proposed a consistent approach for physics, symmetrical to the standard model [(Einstein \& Infeld, 1938). He privileged a continuous field, propagating at light velocity, and leaning upon physical representations of phenomena, before their more precise mathematical description.

«We have two realities: matter and field. ....We cannot build physics on the basis of the matter concept alone. But the division into matter and field is, after the recognition of the equivalence of mass and energy, something artificial and not clearly defined. Could we not reject the concept of matter and build a pure field physics? ... We could regard matter as the regions in space where the field is extremely strong. In this way a new philosophical background could be created.... Only field-energy would be left, and the particle would be merely an area of special density of field-energy. In that case one could hope to deduce the concept of the mass-point together with the equations of the motion of the particles from the field equations- the disturbing dualism would have been removed... One would be compelled to demand that the 
particles themselves would everywhere be describable as singularity free solutions of the completed field-equations. Only then would the general theory of general relativity be a complete theory.... One could believe that it would be possible to find a new and secure foundation for all physics upon the path which had been so successfully begun by Faraday and Maxwell.»

The Einstein's Program has been implicitly supported, and validated, by the International Legal Metrology Organization, by shifting from material standards of space and time, to field standards. In one hand, the velocity of light in vacuum is admitted as a "pure", or primary, fundamental constant in experimental physics, with its numerical value strictly fixed. In another hand, the standard for measures of time is based on the period an electromagnetic wave frequency ( Dimarcq, 2013)

In previous articles (Elbaz, 2012-2015), we showed how the Einstein's program forms a consistent system, beside the standard model, for the universe description. It allows us to complete the universe grasp, like both eyes give us access to tridimensional vision, or both ears to stereophonic audition. Starting from a scalar field propagating at light velocity c, its solutions yield properties of matter and interactions. The standing waves corresponds to matter, and their adiabatic variations to interactions: variations of frequencies to electromagnetism, and variations of velocity of light to gravitation. When the frequencies are infinitely high, and undetectable, defining the geometrical optics approximation, the oscillations are hidden. This leads the field to appear as concentrated, as point-like particles for matter, and as rays for trajectories of matter and of interactions. This leads also to the kinematical and dynamical laws for matter, in classical relativistic and in quantum frameworks. Since high frequencies, leading to point-like particles, are eluded, their kinematical and dynamical properties are expressed by time-like functions only. Their descriptions are then incomplete. This was explicitly emphasized by Einstein in his program, not only for quantum mechanics, but also for general relativity. Consequently, the Einstein's program enables to supplement them with a space-like amplitude function. It leads to the point-like character for a material particle, to the variational least action principle, and to the energy-momentum conservation law, usually admitted independently as fundamental.

In this article, we propose to show how, following the Einstein's program, a scalar field $\varepsilon$ propagating at light velocity c, introduces a space-like amplitude function u, which allows a unified approach of the quantum double slit experiment, both for light and for electrons.

\section{The Einstein's Program}

Since gravity is not directly involved, the velocity of light in vacuum c, is admitted as constant (Elbaz, 2012). We summarize some equations deduced from Einstein's program, in order to show how they are related to the main equations of double slit experiment, otherwise widely documented.

\subsection{Standing Field Kinematics}

Starting from a scalar field $\varepsilon$ propagating at light velocity $\mathrm{c}$, we are assured that its whole following consequences are relativistic. The general harmonic solutions of the d'Alembertian's equation

$$
\square \varepsilon=\Delta \varepsilon-\left(1 / \mathrm{c}^{2}\right)\left(\partial^{2} \varepsilon / \partial \mathrm{t}^{2}\right)=0,
$$

may be reduced to two kinds of elementary ones, according to their kinematic, or their geometric, properties. Progressive waves, like $\cos (\omega t \pm \mathrm{kx})$ with constant frequency $\omega=\mathrm{kc}$, propagate at speed of light in opposite direction. Standing waves, of the form $\varepsilon_{0}\left(\mathrm{x}_{0}, \mathrm{t}_{0}\right)=\mathrm{u}_{0}\left(\mathrm{k}_{0} \mathrm{x}_{0}\right) \psi_{0}\left(\omega_{0} \mathrm{t}_{0}\right)=\cos \left(\omega_{0} \mathrm{t}_{0}\right) \cos \left(\mathrm{k}_{0} \mathrm{x}_{0}\right)$, oscillate locally, as expressed by the separation of variables for space and time. They enable then to define a system of coordinates at rest $\left(\mathrm{x}_{0}, \mathrm{t}_{0}\right)$. As the functions $\mathrm{u}_{0}\left(\mathrm{k}_{0} \mathrm{x}_{0}\right)$ and $\psi_{0}\left(\omega_{0} \mathrm{t}_{0}\right)$ are independent, the frequency $\omega_{0}$ is necessarily constant in $\left(1 / \mathrm{u}_{0}\right) \Delta_{0} \mathrm{u}_{0}=\left(1 / \psi_{0}\right)\left(\partial^{2} \psi_{0} / \mathrm{c}^{2} \partial \mathrm{t}_{0}{ }^{2}\right)=-\mathrm{k}_{0}{ }^{2}=-\omega_{0}{ }^{2} / \mathrm{c}^{2}$. The progressive and standing waves can be considered, either as elementary and basic for the field $\varepsilon$, or as composed from other kinds, since

$$
\begin{aligned}
& \cos \left(\omega_{0} \mathrm{t}_{0}+\mathrm{k}_{0} \mathrm{x}_{0}\right)+\cos \left(\omega_{0} \mathrm{t}_{0}-\mathrm{k}_{0} \mathrm{x}_{0}\right)=2 \cos \left(\omega_{0} \mathrm{t}_{0}\right) \cos \left(\mathrm{k}_{0} \mathrm{x}_{0}\right), \\
& \cos \left(\omega_{0} \mathrm{t}_{0}\right) \cos \left(\mathrm{k}_{0} \mathrm{x}_{0}\right)+\sin \left(\omega_{0} \mathrm{t}_{0}\right) \sin \left(\mathrm{k}_{0} \mathrm{x}_{0}\right)=\cos \left(\omega_{0} \mathrm{t}_{0}-\mathrm{k}_{0} \mathrm{x}_{0}\right)
\end{aligned}
$$

When, in a system of reference $(x, t)$, the frequencies of opposite progressive waves are different

$$
\cos \left(\omega_{1} t-k_{1} x\right)+\cos \left(\omega_{2} t+k_{2} x\right)=2 \cos (\omega t-\beta k x) \cos (k x-\beta \omega t),
$$

with $\beta=\left(\omega_{1}-\omega_{2} / \omega_{1+} \omega_{2}\right)$, and $\omega=\left(\omega_{1+} \omega_{2}\right) / 2=k c$. By identification with (2), they form a standing wave, with main frequency $\omega_{0}$ $=\sqrt{ } \omega_{1} \omega_{2}$ in the system of reference at rest $\left(\mathrm{x}_{0}, \mathrm{t}_{0}\right)$, becoming $\omega$, for the standing wave in motion with a speed $\mathrm{v}$, in the system $(\mathrm{x}, \mathrm{t})$. The correspondence between the systems of reference $\left(\mathrm{x}_{0}, \mathrm{t}_{0}\right)$ and $(\mathrm{x}, \mathrm{t})$, leads to the Lorentz transformation, and to its whole special relativity consequences.

The geometric properties of standing waves at rest, are described by the function of space $\mathrm{u}_{0}\left(\mathrm{k}_{0} \mathrm{x}_{0}\right)$, which obeys the Helmholtz's equation $\Delta_{0} \mathrm{u}_{0}+\mathrm{k}_{0}{ }^{2} \mathrm{u}_{0}=0$. Its solutions verify Bessel spherical functions, and particularly its simplest elementary solution, with spherical symmetry, finite at origin $\mathrm{r}_{0}$ of the reference system, and representing a lumped function, 


$$
\mathrm{u}_{0}\left(\mathrm{k}_{0} \mathrm{r}_{0}\right)=\left(\operatorname{sink}_{0} \mathrm{r}_{0}\right) /\left(\mathrm{k}_{0} \mathrm{r}_{0}\right) \text {, }
$$

In geometrical optics approximation, when the frequency is very high, tending towards infinity $\omega_{0}=\mathrm{k}_{0} \rightarrow \infty$, and then undetectable and eluded, the space function $\mathrm{u}_{0}$ tends towards Dirac's distribution $\mathrm{u}_{0}\left(\mathrm{k}_{0} \mathrm{r}_{0}\right) \rightarrow \delta\left(\mathrm{r}_{0}\right)$. The standing wave of the field behaves as a free classical material particle at rest, isolated in space.

From a kinematical point of view, the central extremum of an extended standing wave, either at rest or in motion, is appropriate to localize its position $\mathrm{x}_{0}$, exactly like the centre of mass for a material system. It verifies, for instance from $(5)$,

$$
\nabla_{0} \mathrm{u}_{0}\left(\mathrm{x}_{0}\right)=0 .
$$

In motion, the Helmholtz's equation, for the amplitude function $u(x, t)$, becomes $\Delta u-\partial^{2} u / c^{2} \partial t^{2}+k_{0}^{2} u=0$. The phase function obeys the equation $\Delta \psi-\partial^{2} \psi / \mathrm{c}^{2} \partial \mathrm{t}^{2}-\mathrm{k}_{0}^{2} \psi=0$. The four-dimensional Minkowski's formalism is useful and appropriate to express invariance properties of standing waves at rest, when they move uniformly. Confirmation is found into invariant quantities obtained from four-quantities, such as coordinates $\mathrm{x}_{\mu} \mathrm{x}^{\mu}=\mathrm{x}_{0}{ }^{2}$ or $\mathrm{x}_{\mu} \mathrm{x}^{\mu}=\mathrm{c}^{2} \mathrm{t}_{0}^{2}$, and functions $\mathrm{u}_{\mu} \mathrm{u}^{\mu}=\mathrm{u}^{2}\left(\mathrm{x}_{0}\right)$ or $\psi_{\mu} \psi^{\mu}=\psi^{2}\left(\mathrm{t}_{0}\right)$. Their space-like or time-like character is absolute, according to the depending coordinate $\mathrm{x}_{0}$ or $\mathrm{t}_{0}$ in the rest system $\left(\mathrm{x}_{0}, \mathrm{t}_{0}\right)$, in which the variables of space and time are separated.

In order to point out the necessarily constant frequency for a standing wave, we precise only that the phase function $\psi$ is a plane wave, leaving the amplitude function $\mathrm{u}(\mathrm{x}, \mathrm{t})$, undetermined inside Helmholtz's equation.

$$
\varepsilon(\omega \mathrm{t}, \mathrm{kx})=\mathrm{u}(\mathrm{kx}, \beta \omega \mathrm{t}) \exp \mathrm{i}(\omega \mathrm{t}-\beta \mathrm{kx}) \quad \varphi=\omega \mathrm{t}-\beta \mathrm{kx},
$$

In special relativity, the equations are based on mass-points, as singularities, moving on trajectories. They lean then directly upon geometrical optics approximation. The periodic equations, generic of standing fields, are hidden. The space coordinates $\mathrm{x}_{\alpha}$, involved in the metric, are point-like dynamical variables, and not field variables $\mathrm{r}$ which would describe an extended repartition in space. Then, for standing waves of a scalar field propagating at light velocity c, with constant frequency $\omega$ and velocity $\mathrm{v}$, the kinematic properties are formally identical with mechanic properties of isolated matter. The Lorentz transformation, and particularly the coefficient $\sqrt{ }\left(1-\beta^{2}\right)$, is specific of standing waves with respect to progressive waves (Elbaz, 1983).

\subsection{Standing Field Dynamics}

All above equations are unlimited with respect to space and time, since $\mathrm{x}$ and t may become infinite. Usually, in order to limit the field, one imposes boundary conditions, in which matter acts either as a source fixing the frequency $\omega$, or as a detector annealing it, as well as a geometrical space boundary fixing the wavelength $\lambda$ through $k=2 \pi / \lambda$. This is not felicitous from relativistic consistency, since space and time operate separately. In addition, matter is physically heterogeneous with respect to field. In order to stay in a homogeneous frame, we rather consider boundaries provided by wave packets. Two progressive waves with different frequencies $\omega_{1}, \omega_{2}$ propagating in the same direction at light velocity, give rise to a wave packet, propagating in the same direction at light velocity, with a main wave with frequency $\omega=\left(\omega_{1+} \omega_{2}\right) / 2$. It is modulated by a wave with frequency $\beta \omega=\left(\omega_{1}-\omega_{2}\right) / 2=\Delta \omega / 2=\Delta \mathrm{kc} / 2$, wavelength $\Lambda=2 \pi / \beta \mathrm{k}$, and period $T=\Lambda / \mathrm{c}$. Since $\beta<1$, the modulation wave acts as an envelope, with space extension $\Delta x=\Lambda / 2$ and and time extension $\Delta \mathrm{t}=\mathrm{T} / 2$, corresponding to the well known Fourier relations $\Delta \mathrm{x} . \Delta \mathrm{k}=2 \pi$ and $\Delta \mathrm{t} . \Delta \omega=2 \pi$.

Then, the Fourier relations represent homogeneous boundary conditions for the scalar field $\varepsilon$. From a physical point of view, they must be associated with the d'Alembertian's equation (1) in order to complete them, emphasizing that the field cannot extend to infinity with respect to space and time.

When the difference of frequencies $\beta \omega=\left(\omega_{1}-\omega_{2}\right) / 2=\Delta \omega / 2<<\omega$ is very small, it can be considered as a perturbation with respect to the main frequency, $\beta \omega=\delta \omega$. Then a wave packet can be assimilated to a progressive monochromatic wave with frequency $\Omega=\omega \pm \delta \omega$, inside the limits fixed by the component frequencies $\omega_{1}=\omega+\delta \omega$ and $\omega_{2}=\omega-\delta \omega$. By difference with standing waves frequencies, which must be constant and monochromatic, progressive fields solutions of (1), may be more complex, with frequencies varying with space and time. An almost monochromatic wave is characterized by a frequency $\Omega(\mathrm{x}, \mathrm{t})$, varying very slowly around a constant $\omega$

$$
\Omega(\mathrm{x}, \mathrm{t})=\mathrm{K}(\mathrm{x}, \mathrm{t}) \mathrm{c}=\omega \pm \delta \Omega(\mathrm{x}, \mathrm{t}) \quad \delta \Omega(\mathrm{x}, \mathrm{t})<<\omega \quad \omega=\text { constant } .
$$

From a physical point of view, we recognize the definition of an adiabatic variation for the frequency (Landau \& Lifchitz, 1960). We can then expect that all following properties of almost fields, occur inside such a process. Instead of admitting a constant frequency $\omega$ of elementary waves propagating all over space-time as given data, we rather consider that it represents the mean value, all over the field, of different slowly varying frequencies $\Omega(\mathrm{x}, \mathrm{t})$. In other words, the modulation waves with perturbation frequencies $\delta \Omega(\mathrm{x}, \mathrm{t})$, propagating at light velocity, behave as interactions between main waves, leading their mean frequency $\omega$ to remain practically constant, all over the space-time (Elbaz, 2014,2015).

From a mathematical point of view, almost fields properties derive from monochromatic ones, through the variation of 
constants method (Duhamel principle). For an almost standing wave, equation (7) becomes

$$
\varepsilon(\mathrm{x}, \mathrm{t})=\mathrm{U}(\mathrm{x}, \mathrm{t}) \exp \mathrm{i} \phi(\mathrm{x}, \mathrm{t}) \quad \phi(\mathrm{x}, \mathrm{t})=\Omega(\mathrm{x}, \mathrm{t}) \mathrm{t}-\mathrm{K}(\mathrm{x}, \mathrm{t}) \mathrm{x}+2 \mathrm{n} \pi,
$$

where second order products $\delta \Omega \mathrm{dt} \approx 0$ and $\delta \mathrm{K} . \mathrm{dx} \approx 0$, defined modulo $2 \pi$, are neglected at first order of approximation. This is equivalent to incorporate directly, in almost monochromatic solutions, the boundary conditions defined by the Fourier relations.

$$
\mathrm{d} \phi(\mathrm{x}, \mathrm{t})=\Omega(\mathrm{x}, \mathrm{t}) \mathrm{dt}-\mathrm{K}(\mathrm{x}, \mathrm{t}) . \mathrm{dx} \approx \omega \mathrm{dt}-\mathrm{k} \cdot \mathrm{dx} . \quad \mathrm{U}(\mathrm{x}, \mathrm{t})=\mathrm{u}(\mathrm{x}, \mathrm{t}) \pm \delta \mathrm{U}(\mathrm{x}, \mathrm{t})
$$

Following $\partial^{\mu} \partial_{\mu} \varepsilon=0$ in (1), which becomes $\partial^{\mu} \partial_{\mu} \varepsilon(x, t)=0$, from (9) we obtain,

$$
\begin{array}{cl}
\partial^{\mu} \partial_{\mu} \mathrm{U}-\mathrm{U} \partial^{\mu} \phi \partial_{\mu} \phi=0 \text { or } & \partial^{2} \mathrm{U} / \mathrm{c}^{2} \partial \mathrm{t}^{2}-\nabla^{2} \mathrm{U}-\mathrm{U}\left[(\partial \phi / \mathrm{c} \partial \mathrm{t})^{2}-(\nabla \phi)^{2}\right]=0 \\
\partial^{\mu}\left(\mathrm{U}^{2} \partial_{\mu} \phi\right)=0 & \text { or } \quad \partial\left(\mathrm{U}^{2} \Omega\right) / \mathrm{c}^{2} \partial \mathrm{t}+\nabla \cdot\left(\mathrm{U}^{2} \beta \mathrm{K}\right)=0
\end{array}
$$

These relations apply to progressive waves for $\beta= \pm 1$, to standing waves at rest for $\beta=0$ and in motion for $\beta<1$, to monochromatic waves for $\omega$ and $\mathrm{k}$ constant, to almost monochromatic waves for varying $\Omega(\mathrm{x}, \mathrm{t})$ et $\mathrm{K}(\mathrm{x}, \mathrm{t})$. They yield dynamical properties for energy-momentum conservation, and variational least action principle, both for standing fields and for almost standing fields.

For a standing wave with constant frequency either at rest or in motion, $\delta \Omega(\mathrm{x}, \mathrm{t})=0$, so that $(12)$ reduces to

$$
\partial \mathrm{u}_{0}{ }^{2} / \partial \mathrm{t}_{0}=0 \quad \partial \mathrm{u}^{2} / \partial \mathrm{t}+\nabla \cdot \mathrm{u}^{2} \mathrm{v}=0 \quad \text { or } \quad \partial_{\mu} \mathrm{w}^{\mu}=0
$$

where $\mathrm{w}^{\mu}=\left(\mathrm{u}^{2}, \mathrm{u}^{2} \mathrm{v} / \mathrm{c}\right)=\mathrm{u}_{0}\left(\mathrm{x}_{0}\right)^{2}(1, \mathrm{v} / \mathrm{c}) / \sqrt{ }\left(1-\beta^{2}\right)$ is a four-dimensional quantity. This continuity equation for $\mathrm{u}^{2}$, is formally identical with Newton's continuity equation for matter and its momentum densities

$$
\partial \mu / \partial \mathrm{t}+\nabla \cdot \mu \mathrm{v}=0 \quad \text { with } \quad \mathrm{u}^{2}=\mu \mathrm{c}^{2} .
$$

We are led to admit, by transposition, that $\mathrm{u}^{2}$ represents the energy density of the standing field.

Following relations (5) and (6) in the spherical symmetry case, for its kinematical behavior, the space function $\mathrm{u}_{0}$ can be reduced to its point-like centre of energy density. Following (6), its position $\mathrm{x}_{0}$ is such that

$$
\nabla_{0} \mathrm{u}_{0}^{2}=0 \quad \nabla \mathrm{u}^{2}+\left(\partial \mathrm{u}^{2} \mathrm{v} / \mathrm{c}^{2} \partial \mathrm{t}\right)=0 \quad \nabla \times \mathrm{v}=\quad \text { or } \quad \pi^{\mu v}=\partial^{\mu} \mathrm{w}^{v}-\partial^{v} \mathrm{w}^{\mu}=0,
$$

Since $u^{2}$ is a standing wave energy density spread in space, and then a potential energy density, $F=-\nabla u^{2}=-\nabla w_{p}$ is a force density, and $\partial \mathrm{u}^{2} \mathrm{v} / \mathrm{c}^{2} \partial \mathrm{t}$ a density momentum, while $\pi^{\mu \nu}$ is a four-dimensional force density.

Equation (15), in which energy density $\mathrm{w}^{\mu}$ is a four-dimensional gradient $\partial^{\mu} \mathrm{a}$, is mathematically equivalent to the least action relation

$$
\delta \int \mathrm{da}=0 \quad \delta \int \partial^{\mu} \mathrm{adx}_{\mu}=0 \quad \text { with } \quad \mathrm{w}^{\mu}=\partial^{\mu} \mathrm{a} .
$$

When we transpose the mass density $\mu=\mathrm{u}^{2} / \mathrm{c}^{2}$, and take into account identities the $\nabla \mathrm{P}^{2}=2(\mathrm{P} . \nabla) \mathrm{P}+2 \mathrm{P} \times(\nabla \times \mathrm{P})$ and $\mathrm{dP} / \mathrm{dt}=$ $\partial \mathrm{P} / \partial \mathrm{t}+(\mathrm{v} . \nabla) \mathrm{P}$ for $\mathrm{c}$ and $\mathrm{v}$ constant, after integration with respect to space, we get the equation for matter

$$
\mathrm{dp} / \mathrm{dt}=-\nabla \mathrm{mc}^{2}+\left\{\nabla(\mathrm{mv})^{2}\right\} / 2 \mathrm{~m} \quad \mathrm{dp} / \mathrm{dt}=\nabla \mathrm{L}_{\mathrm{m}}=-\nabla \mathrm{m}_{0} \mathrm{c}^{2} \sqrt{ }\left(1-\beta^{2}\right) .
$$

We retrieve the relativistic Lagrangian of mechanics, usually admitted for free matter $L_{m}=-m_{0} c^{2} \sqrt{ }\left(1-\beta^{2}\right)$.

\subsection{Electromagnetic Interaction}

For of an almost standing wave, the continuity equation relates to the total energy density, $\mathrm{W}=\mathrm{U}^{2} \Omega=\mathrm{W}+\delta \mathrm{W}$, sum of the mean standing wave $\mathrm{w}$ and of the interactions $\delta \mathrm{W}$. Equation (15) becomes

$$
\Pi^{\mu \nu}=\partial^{\mu} \mathrm{W}^{\nu}-\partial^{v} \mathrm{~W}^{\mu}=0 \quad \text { or } \quad \Pi^{\mu \nu}=\pi^{\mu \nu}+\delta \Pi^{\mu \nu}=0
$$

By difference with the null four-dimensional density force $\pi^{\mu v}$ for a standing wave, only the total density force $\Pi^{\mu v}$ for an almost standing wave vanishes. In the first case, this asserts the space stability of an isolated standing wave, while in the second case, the space stability concerns the whole almost standing wave. It behaves as a system composed of two sub-systems, the mean standing field with high frequency $\Omega(\mathrm{x}, \mathrm{t}) \approx \omega$, and the interaction field with lower frequency $\delta \Omega(\mathrm{x}, \mathrm{t})$, each one exerting an equal and opposite density force $\pi^{\mu \nu}=-\delta \Pi^{\mu \nu}$ against the other.

In (15), the vanishing four-dimensional force density tensor $\pi^{\mu v}$ of a standing wave, asserts that the energy-momentum density four-vector $\mathrm{w}^{\mu}$ is four-parallel, or directed along the motion velocity $\mathbf{v}$. By comparison, for an almost standing wave, the total energy-momentum density tensor $\Pi^{\mu v}$ which still vanishes, asserts also that the total energy-momentum density four-vector $\mathrm{W}^{\mu}$ is four-parallel, or directed along the motion velocity v. However, the mean energy-momentum density tensor $\pi^{\mu \nu}$, no longer vanishes in (18) as previously in (15): the mean energy-momentum density four-vector $\mathrm{w}^{\mu}$ is then no longer parallel. This comes from the opposite density force $\delta \Pi^{\mu v}$ exerted by the interaction.

It appears that an almost standing field behaves as a whole system in motion which can be split into two sub-systems: the mean standing field and the interaction field. Both are moving with velocity $\mathrm{v}$, while exerting each other opposite 
forces in different directions, including perpendicularly to the velocity v. The perturbation field, arising from local frequency variations $\delta \Omega(\mathrm{x}, \mathrm{t})$, introduces orthogonal components in interaction density force and momentum, with regard to the main constant frequency $\omega$ of the plane wave (7).

Relations (17), generalized by constants variation method for mass $M(x, t)=m \pm \delta M(x, t)$, become

$$
\nabla \mathrm{Mc}^{2}+\partial \mathrm{P} / \partial \mathrm{t}=0 \quad \nabla \times \mathrm{P}=0 \quad \mathrm{dP} / \mathrm{dt}=-\nabla \mathrm{Mc}^{2}+\left(\nabla \mathrm{P}^{2}\right) / 2 \mathrm{M} .
$$

The non vanishing density force $\delta \Pi^{\mu v} \neq 0$ exerted by the interaction is formally identical with the electromagnetic tensor $\mathrm{F}^{\mu \nu}=\partial^{\mu} \mathrm{A}^{v}-\partial^{v} \mathrm{~A}^{\mu} \neq 0$. We can set them in correspondence $\delta \Pi^{\mu v}=\mathrm{eF}^{\mu \nu}$, through a constant charge e, in which $\delta \mathrm{M}(\mathrm{x}, \mathrm{t})=$ $\mathrm{eV}(\mathrm{x}, \mathrm{t}) / \mathrm{c}^{2}$ and $\delta \mathrm{P}(\mathrm{x}, \mathrm{t})=\mathrm{eA}(\mathrm{x}, \mathrm{t}) / \mathrm{c}$. The double sign for mass variation corresponds to the two signs for electric charges, or to emission and absorption of electromagnetic energy by matter. We retrieve the minimum coupling of classical electrodynamics, $\mathrm{P}^{\mu}(\mathrm{x}, \mathrm{t})=\mathrm{p}^{\mu}+\mathrm{e} \mathrm{A}^{\mu}(\mathrm{x}, \mathrm{t}) / \mathrm{c}$, with $\mathrm{M}(\mathrm{x}, \mathrm{t}) \mathrm{c}^{2}=\mathrm{mc}^{2}+\mathrm{eV}(\mathrm{x}, \mathrm{t})$, and $\mathrm{P}(\mathrm{x}, \mathrm{t})=\mathrm{p}+\mathrm{eA}(\mathrm{x}, \mathrm{t}) / \mathrm{c}$ where electromagnetic energy-momentum exchanged with a particle, is very small with respect to its own energy-momentum eA ${ }^{\mu}(x, t) / c=$ $\delta \mathrm{P}^{\mu}(\mathrm{x}, \mathrm{t})<<\mathrm{p}^{\mu}$. Electromagnetic interaction is then directly linked to frequencies variations of the field $\varepsilon$ (Landau $\&$ Lifchitz 1962).

Accordingly, the relativistic Newton's equation for charged matter, with the Lorentz force, derives from (19)

$$
\mathrm{dP} / \mathrm{dt}=-\nabla \mathrm{m}_{0} \mathrm{c}^{2} \sqrt{ }\left(1-\beta^{2}\right)+\mathrm{e}(\mathrm{E}+\mathrm{v} \times \mathrm{H} / \mathrm{c}) .
$$

\subsection{Adiabatic Invariance}

For an almost standing wave, we get from (11), in first order approximation,

$$
\left[\partial \mathrm{U}^{2} / \partial \mathrm{t}+\nabla \cdot \mathrm{U}^{2} \mathrm{v}\right] / \mathrm{U}^{2}+\delta[\partial \Omega / \partial \mathrm{t}+\nabla \cdot \Omega \mathrm{v}] / \Omega=0 \quad \text { or }\left(\partial_{v} \mathrm{~W}^{v}\right) / \mathrm{W}+\delta\left(\partial_{v} \Omega^{v}\right) / \Omega=0 .
$$

with energy density $\mathrm{W}=\mathrm{W} \pm \delta \mathrm{W}=\mu \mathrm{c}^{2}=\mu \mathrm{c}^{2} \pm \delta \mu \mathrm{c}^{2}$, four-dimensional energy density $\mathrm{W}^{v}=\mathrm{w}^{v} \pm \delta \mathrm{W}=\left(\mu \mathrm{c}^{2}, \mu \mathrm{vc}\right)$, frequency $\Omega=\omega \pm \delta \Omega$, and four-dimensional frequency $\Omega^{v}=(\Omega, \Omega v / c)$, leading to

$$
\mathrm{W}=\mathrm{I} \Omega \quad \text { and } \quad \mathrm{W}^{\mathrm{v}}=\mathrm{I} \Omega^{\mathrm{v}}
$$

when we take into account the double sign in frequency variation $\delta \Omega$. The constant I is an adiabatic invariant density. In first approximation, they reduce to energy-momentum densities, and to their variations, relations

$$
\begin{array}{ccc}
\mathrm{W}^{v}=\mathrm{I} \omega^{v} & \text { or } \quad \mu \mathrm{c}^{2}=\mathrm{I} \omega \text { and } \mu \mathrm{v}=\mathrm{I} \beta \mathrm{k} \\
\delta \mathrm{W}^{v}=\mathrm{I} \delta \Omega^{v} & \text { or } & \delta \mu \mathrm{c}^{2}=\mathrm{I} \delta \Omega \quad \text { and } \delta \mu \mathrm{v}=\mathrm{I} \delta \beta \mathrm{K}
\end{array}
$$

Integrations of $\mu$ and I densities with respect to space, lead to relations between four-energy and four-frequency through the adiabatic invariant $\mathrm{H}$, formally identical with the Planck's constant $\mathrm{h}$.

$$
\mathrm{E}^{\mathrm{v}}=\left(\mathrm{mc}^{2}, \mathrm{pc}\right)=\mathrm{m}_{0} \mathrm{c}^{2} \mathrm{u}^{\mathrm{v}}=\mathrm{H} \omega^{\mathrm{v}}=\mathrm{H}(\omega, \mathrm{kc}) \quad \mathrm{m}_{0} \mathrm{c}^{2}=\mathrm{H} \omega_{0}
$$

The adiabatic variations frequency $\delta \Omega$ for the standing wave $\Omega$ corresponding to matter, lead to electromagnetic interaction constituted by progressive waves. Electromagnetic interaction energy derives from mass variation $\mathrm{dE}=\mathrm{c}^{2} \mathrm{dm}$, leaning directly to the wave property of matter: its energy $\mathrm{dE}=\mathrm{hdv}=\mathrm{c}^{2} \mathrm{dm}$ derives from variations of matter energy $\mathrm{E}=\mathrm{h} v=\mathrm{mc}^{2}$.

\subsection{Remarks}

We notice that the double frequencies, $\Omega \approx \omega$ and $\delta \Omega<<\omega$ of an almost standing wave, enables it to verify simultaneously geometrical optics approximation, when experimental boundary conditions are such that, they are very large regard to its main wavelength $\lambda=2 \pi \mathrm{c} / \omega$, and of same order of its perturbation wavelength $\Lambda=2 \pi \mathrm{c} / \Omega$. In that case the particle and the wave behaviors coexist.

\section{Application to Double Slit Experiment}

The Einstein's program offers a tool for a unified approach, and treatment, of the double slit experiment, for matter and light, in their classical, and quantum, manifestations.

\subsection{Classical Physics}

The specific phenomenon, displayed by the double-slit experiment, is characterized by interference pattern with bright and dark bands on the screen. It is specific of the wave behavior of light, exhibited when it passes through the slits.

The holes must be sufficiently small, of the order of some wavelengths. They behave as secondary sources, enabling the light to be diffracted as wavelets, with a definite phase relationship between them. Such a condition is usually obtained with a small extended source for light. It behaves then as the centre of spherical light waves, able to interfere coherently with themselves. In classical physics, since we do not have access to a model of atom as light source, the emitted wavelength $\lambda_{\mathrm{k}}=2 \pi / \mathrm{k}_{\mathrm{k}}$, is admitted as given data. The distance between the light source and the slits is very much larger 
than the wavelength. This, it corresponds to the geometrical optics condition $\lambda \rightarrow 0$. Experimentally, the light propagates along rays, which can be assimilated to point trajectories.

Because of the static character of boundaries, only the time independent part $\mathrm{u}(\mathrm{kx})$, of the propagation equation $\square \varepsilon=\Delta \varepsilon$ $-\left(1 / \mathrm{c}^{2}\right)\left(\partial^{2} \varepsilon / \partial \mathrm{t}^{2}\right)=0$, is useful to determine the geometrical repartition of the light in space. It verifies the Helmholtz's equation $\Delta \mathrm{u}+\mathrm{k}_{\mathrm{k}}{ }^{2} \mathrm{u}=0$. Its elementary spherical wavelets solutions verify, either the imaginary form $\mathrm{e}^{\mathrm{ikr}} / \mathrm{r}$, or the real one, $\mathrm{u}_{\mathrm{k}}\left(\mathrm{k}_{\mathrm{k}} \mathrm{r}\right)=\left(\sin _{\mathrm{k}} \mathrm{r}\right) /\left(\mathrm{k}_{\mathrm{k}} \mathrm{r}\right)$, with origins defined by the positions of the point-like source and slits, as boundaries conditions. The following mathematical solutions for the double slit experiment are otherwise well documented.

We retain that, from physical and mathematical points of view, the continuous second order partial differential Helmholtz's equation conciliates both characters of light, disclosed by the double-slit experiment: its particle kinematic behavior, in geometrical optics approximation where travelled distances are much larger than the wavelength, and its wave kinematic behavior, in wave optics approximation where boundaries are of order of the wavelength. The timeindependent character of the Helmholtz's equation is consistent with, or expresses, the appearance, at one and same time, of the interference pattern extended in space.

\subsection{Quantum Mechanics}

\subsubsection{Experiment with Light}

All above considerations remain unchanged in quantum mechanics, except that the interference pattern extended in space, does not appear directly and simultaneously. It reveals progressively in time, following the juxtaposition of successive impacts due to point-like individual photons.

Experimentally, in order to realize the double slit quantum experiment, one may maintain geometrically all classical material wave conditions. Only the intensity of the emitted light requires to be sufficiently reduced, until reaching level of energy quantum $\mathrm{E}=\mathrm{h} v$.

Mathematically, passing from the previous geometrically extended classical solution of the Helmholtz's equation $\Delta \mathrm{u}+\mathrm{k}_{\mathrm{k}}{ }^{2} \mathrm{u}=0$, to a point-like localization of an individual photon, arises with the introduction of Planck's constant $\mathrm{h}$, with Heisenberg relations..

Physically, such a passage is admitted as representing a collapse. It occurs from an extended, to a concentrated, energy repartition in space, following the Heisenberg-von Neumann postulate in quantum mechanics. It implies that the wave and the particle behaviors of light must be considered on an equal footing, as solutions of the propagation equation. Nevertheless, both aspects do not appear simultaneously: the wave aspect during the motion and before the observation, the particle aspect after the emission and the detection. Then, such an energy collapse for light, implies that a photon is usually observable only when it disappears. Nevertheless, S. Haroche and his collaborators, has realized "a new type of atomic detector, able to record the trace of a single photon, without absorbing energy» (Haroche, S., 2007).

\subsubsection{Experiment with Matter}

In the quantum mechanics framework, the same kind of double-slit experiment can be performed with electrons, leading to the same kind of interference patterns. It shows that matter exhibits wave-duality behavior, exactly like light (Merli, Missiroli, Pozzi, 1976) (Rosa, 2012). In order to describe it, the d'Alembertian's equation of propagation $\square \varepsilon=0$, is then replaced by the non relativist Schrödinger equation (ih/2 $\pi) \partial \psi / \partial \mathrm{t}+\left(\mathrm{h}^{2} / 8 \pi^{2} \mathrm{~m}\right) \Delta \psi=0$. The energy involved is the kinetic energy of the particle $E_{k}=p^{2} / 2 m$, and not its main energy $E=m c^{2} \approx E_{k}+E_{0}$. The mass of electron $m_{0}=E_{0} / c^{2}$ is eluded, or hidden, in the whole process. Consequently, one admits that it remains constant and unaffected by the motion. In such a framework, it is admitted that an electron behaves always as a particle, and never as a wave. Only its point-like position is governed by a wave-like function with the Schrödinger equation. Consequently, the space extension $\Delta \mathrm{x}$ of the Heisenberg relation represents the interval inside which the probabilistic point-like position $\mathrm{x}$ may be found.

The mass of electron $\mathrm{m}_{0}$ does not absorb or emit kinetic energy, which constitutes an external physical entity. Such a non relativist treatment process, implies that the physical nature of the electron mass-energy $\mathrm{E}=\mathrm{mc}^{2}$, and of its kinetic energy $E_{k}=p^{2} / 2 m$, are distinct and independent.

The Schrödinger equation admits only implicitly the point-like character of an electron. In order to get its experimental explicit point-like character, it needs to be supplemented by an extraneous condition. It is mathematically attributed to the Heisenberg relations, and physically to the measure, at origin of the collapse. The Schrödinger equation describes the electron motion with speed $\mathrm{v}$, with a second order partial differential equation with respect to space, which characterizes mathematically a wave. Such a property appears when the boundary conditions are of order of the Broglie's wavelength $\lambda_{b}=2 \pi / \mathrm{k}=\mathrm{h} / \mathrm{mv}$, equivalent to the Heisenberg relations, particularly in the especially arranged double-slit experimental conditions. Their static character leads to retain the Helmholtz's equation $\Delta u+k_{b}{ }^{2} u=0$. It derives from the non relativist Schrödinger's propagation equation. It yields interference patterns obtained with electrons. The space periodicity is fixed by de Broglie's wavelength. 
In quantum mechanics, the $\psi$ function represents, either a wave function associated with all its possible positions, or a quantum state occurring within all possible others, inside the limits fixed by the Heisenberg relations. Its effective realization, either through a measured position, or its quantum state, appears then as a collapse, with regard to other numerous solutions of $\psi$. It occurs at the end of the process, expressed when an experimental measurement is made. When, following quantum mechanics historical elaboration, we keep close to the experimental evidence, we have access to an electron only at emission with quantified energy hv, and at detection as a localized particle, and not during its propagation in space wherever between. The collapse describes then a probabilistic event. Such a conclusion leans upon the particle point of view admitted all along the quantum process.

\subsection{Hidden Variables}

As well known, Einstein was unsatisfied by such a probabilistic approach. He attributed it to a lack of knowledge. «The statistical character of the present theory would then have to be a necessary consequence of the incompleteness of the description of the systems in quantum mechanics." Beyond him, since the 1920 years, numerous works were devoted to the problem of hidden variables. In general, they concern the position, and not the description, nor the dimension, of an electron as a fundamental material particle. They hold inside the framework of the non-relativist Schrödinger equation, or its equivalent fundamental formulations.

For instance, instead of waiting for the collapse to find the experimental position of the particle, Bohm introduced it from the beginning, as a hidden variable $\mathrm{x}(\mathrm{t})$ of the usual Schrödinger equation. From its solution $\psi=\mathrm{a} . \operatorname{expi} 2 \pi \mathrm{S} / \mathrm{h}$, the nonlocal quantum potential $\mathrm{Q}=-\left(\mathrm{h}^{2} \nabla^{2} \mathrm{a}\right) / 8 \pi^{2} \mathrm{a}$, guides the motion of the particle, whose trajectory verifies $\mathrm{dx}(\mathrm{t}) / \mathrm{dt}=\mathrm{h} \nabla \mathrm{S} / 4 \pi \mathrm{m}$. (Bohm, 1952)

The introduction of the mass-energy $\mathrm{E}_{0}=\mathrm{m}_{0} \mathrm{c}^{2}$, in relativist quantum mechanics, completes the non relativist Schrödinger equation, or its equivalent formulations of quantum mechanics. It yields the Dirac's equation for a single particle, with coming out of the spin as a new physical property, and spinors as mathematical expressions. Consequently, in quantum field theories, like quantum electrodynamics, a particle can, not only be created or annihilated, but it is no longer single and isolated.

Nevertheless, in all cases, the quantum expression of material particles properties remains incomplete, since the fundamental equations are time-like. The absolute, and independent, time-like or space-like characters, leave open the geometrical description, and extension, in quantum mechanics: it can be point-like, and represented by a Dirac's space-like distribution $\delta\left(\mathrm{r}_{0}\right)$, or extended following, and consistent with, light-like interactions.

As shown above, for the Einstein's program, such a concentrated distribution appears only as the geometrical optics approximation of a more general space-like amplitude function $\mathrm{u}(\mathrm{x}, \mathrm{t})$. It completes the quantum framework, based upon time-like equations: of Klein-Gordon, (yielding the non-relativist Schrödinger equation), for bosons, and of Dirac (yielding half-integer spins), for fermions.

\subsection{Einstein's Program Applications}

In addition to introduce a space-like function for material particles, supplementing time-like functions involved in quantum field theory, the Einstein's program tends towards a theoretical economy by showing how the different properties exhibited by matter and light in double slit experiment, derive from a basic scalar field propagating at velocity of light $\mathrm{c}$.

\subsubsection{Theoretical Economy}

Following the Einstein's program, the same Helmholtz's equations describe the space repartition of light and matter. In the double slit experiments, one must specify the wave numbers $\mathrm{k}_{\mathrm{k}}=2 \pi / \lambda_{\mathrm{k}}=2 \pi \mathrm{c} / v_{\mathrm{k}}$ for light with frequency $v_{\mathrm{k}}$, and $\mathrm{k}_{\mathrm{b}}=2 \pi / \lambda_{\mathrm{b}}=2 \pi \mathrm{mv} / \mathrm{h}$ for matter with mass $\mathrm{m}$, in addition to boundary conditions existing for space. In both cases, the corresponding Helmholtz's equations derive from suitable approximations of the d'Alembertian's equation $\square \varepsilon=0$, for a scalar field propagating at speed of light $\mathrm{c}$.

Thus, matter and light remain always physically and mathematically closely linked,all along their manifestations, not only at their emission and detection, but also along their travel, by interactions with boundaries

The Einstein's program enables a theoretical economy by deriving, such a close link according to the properties of the amplitude function $U$ of an almost standing field. Following (11), relations (23) (24) establish the adiabatic invariant I, leading formally to Planck's constant h. It links energy-momentum densities $\mathrm{w}^{v}=\mathrm{I} \omega^{v}$ for the main field, and $\delta \mathrm{W}^{v}=\mathrm{I} \delta \Omega^{v}$ for the interaction field. By transposition, and integration, they correspond respectively to the second quantification for matter, and to the first quantification for light. It is well known that, historically, more than two decades separated their discoveries: by Planck and Einstein for particles of light, and by de Broglie, Schrödinger, Heisenberg, for particles of matter. 


\subsubsection{Wave-particle Duality}

In the non relativist quantum mechanics framework, it is admitted that an individual particle, like an electron or a photon, holds continuously its point-like character from its emission by a localized source, to its local detection. During its travel, it cannot be destroyed and re-created. Thus, its physically possible opposite behaviors, either as an extended wave or as a concentrated particle, according boundary conditions, has intrigued physicists since 1920 years. How a point-like particle, could be aware of remote physical boundaries, in order to adapt its motion following a wave repartition, particularly in a the double-slit experiment?

For instance, and as illustration, many experiments were devoted to try to determine through which slit the particle had effectively traveled?

The Einstein's program invites us to adopt an opposite point of view, by dealing with wave field all along the process. It emphasizes the structural role of standing solutions of the scalar field propagating at light velocity for matter.

Theoretically, they define rest systems, such the laboratory system. They yield special relativist properties for matter, particularly through the Lorentz transformation and its numerical coefficient, with energy conservation law and least action principle.

Experimentally, physical devices rely on matter. The phenomenological boundary conditions for matter show that it is locally concentrated in atoms. This justifies that the geometrical optics approximation for the spherical standing waves energy, applies for electrons and for light, both at emission and detection from atoms. Depending their geometrical extension in double-slits experiments, they determine wave or particle manifestations, for matter first, and consequently for light, acting as its relativist main energy perturbation.

For matter and for light, a unique Helmholtz's equation describes the whole results of its motion. Where boundary conditions are much larger than the wavelength $\lambda$, like the distance $L$ between the source and the slits $L>>\lambda$, fulfilling the geometrical optics approximation condition $\lambda \rightarrow 0$, either for the light, or for the electron in double slit experiments, they behave as particles moving along trajectories or rays. Where boundary conditions are of order of the wavelength, like at the slits $\mathrm{L} \approx \lambda$, fulfilling the wave optics approximation condition, they lead to wave propagation.

\section{References}

Bohm, D. (1952). A Suggested Interpretation of the Quantum Theory in Terms of 'Hidden' Variables, I and II. Physical Review, 85, 166-193. http://dx.doi.org/10.1103/PhysRev.85.166

Dimarcq, N. (2013). La mesure du temps. Planete astronomie. http://www.planetastronomy.com/special/2014-special/05nov/Dimarcq-IAP.htm

Dirac, P. A. M. (1963). The Evolution of the Physicist's Picture of Nature. Scientific American, may 1963. http://dx.doi.org/10.1038/scientificamerican0563-45

Einstein, A. (1905). Concerning an Heuristic Point of View toward the Emission and Transformation of Light, Ann. Phys., 17, 132 Translation into English American Journal of Physics, 33(5).

Einstein, A. (1949). Albert Einstein: Philosopher, Scientist. Cambridge University Press, London, 2, 667.

Einstein, A., \& Infeld, L. (1938). The Evolution of Physics. Cambridge University Press, Cambridge, 29, 256.

Elbaz, C (2015). On Einstein's Program and Quantum Mechanics, Applied Physics Research, 7(6), http://dx.doi.org/10.5539/apr.v7n6p126

Elbaz, C. (1983). L'onde stationnaire et la transformation de Lorentz, C.R. Acad, Sc, Paris, 298(13), 543.

Elbaz, C. (2012). Gravitational and electromagnetic properties of almost standing fields, Discrete and Continuous Dynamical Systems, A.I.M.S, Series B, 17, 835-849.

Elbaz, C. (2013). Sur les programmes d'Albert Einstein et de Louis de Broglie. Une contribution. Annales Fondation Louis de Broglie, 38, 195-217.

Elbaz, C. (2014). Wave-particle duality in Einstein-de Broglie programs. Journal of Modern Physics, 5, 2192-2199. http://dx.doi.org/10.4236/jmp.2014.518213

Englert, Berthold-Georg (1996). Fringe Visibility and Which-Way Information: An Inequality. Phys. Rev. Lett., 77(11), 2154-2157. http://dx.doi.org/10.1103/PhysRevLett.77.2154

Feynman, R. P., Leighton R. B., \& Sands. M. (1965). The Feynman Lectures on Physics, 3. US: Addison-Wesley, 1.11.8. ISBN 0201021188.

Greenberger, D. M., \& Yasin, A. (1988). Simultaneous wave and particle knowledge in a neutron interferometer. Phys. Lett. A, 128(8), 391-394. http://dx.doi.org/10.1016/0375-9601(88)90114-4 
Haroche, S. (2007). Vie et mort d'un photon : une autre manière de voir, Lettre du collège de France, 20.

Landau, L., \& Lifchitz, E. (1960). Mechanics, Pergamon, 154.

Landau, L., \& Lifchitz, E. (1962). The Classical Theory of Fields, Pergamon, 102.

Merli, P. G., Missiroli, G. F., \& Pozzi, G. (1976). On the statistical aspect of electron interference phenomena. American Journal of Physics, 44, 306-307. http://dx.doi.org/10.1119/1.10184

Rosa, R. (2012). The Merli-Missiroli-Pozzi Two-Slit Electron-Interference Experiment. Physics in Perspective, 14, 178-195. http://dx.doi.org/10.1007/s00016-011-0079-0

\section{(cc) BY}

This work is licensed under a Creative Commons Attribution 3.0 License. 